\title{
Effects of L-carnitine on Polycystic Ovary Syndrome
}

\author{
Saghar Salehpour ${ }^{1}$, Leila Nazari ${ }^{1}$, Sedighe Hoseini ${ }^{1}$, Parya Bameni Moghaddam¹, Latif Gachkar ${ }^{1}$
}

${ }^{1}$ Department of Obstetrics and Gynecology, Preventative Gynecology Research Center, Shahid Beheshti University of Medical Sciences, Tehran, Iran

\begin{abstract}
Objective: Polycystic ovary syndrome (PCOS) is a common disorder in women of reproductive age. This study investigated the effects of L-carnitine on the clinical and laboratory findings of women with PCOS.

Methods: Eighty women diagnosed with PCOS between 2017 and 2018 by the Rotterdam Criteria were enrolled in the study; six were lost during the study. The participants were given L-carnitine $3 \mathrm{~g}$ daily (Pursinapharma, Iran) for three months. Blood samples were taken after overnight fasting at baseline and three months into the study to assess the levels of fasting glucose, insulin, triglycerides, high-density lipoprotein (HDL), low-density lipoprotein (LDL), free testosterone, dehydroepiandrosterone (DHEA), and the insulin resistance index (HOMA-IR). The patients were weighed before and after treatment and had their body mass index (BMI) calculated. Menstrual cycles and manifestations of hirsutism were also assessed.

Results: The data showed a significant improvement in insulin sensitivity and decreases in serum LDL levels and the BMI after three months of treatment. There was a significant increase in serum HDL levels. More regular menstrual cycles and decreased hirsutism were also observed.

Conclusion: It appears that treatment with L-carnitine might decrease the risk of cardiovascular events by normalizing metabolic profiles and the BMI.
\end{abstract}

Keywords: polycystic ovary syndrome, L-carnitine, hyperinsulinemia, HOMA index

\section{INTRODUCTION}

Polycystic ovary syndrome (PCOS) is a common disorder that affects $15-20 \%$ of women of reproductive age. The characterization of PCOS may require ultrasound examination and the observation of other signs such as irregular menstrual cycles, hyperandrogenism leading to acne, alopecia, hirsutism, insulin resistance, dyslipidemias, android obesity, early pregnancy loss, and infertility (Raja-Khan et al., 2011; Stener-Victorin et al., 2013; Marshall \& Dunaif, 2012).

Despite the relevance and prevalence of PCOS, there is no consensus on how the condition should be treated and managed. Different results have been reported from treatment protocols using metformin and statins. Treatments with myo-inositol and $\mathrm{N}$-acetylcysteine have been recently described. Several methods have been used to treat women with PCOS, but there is no agreement on which is the most effective. These points stress the need for more research and studies on the matter (Banaszewska et al., 2009; 2011; Kumar et al., 2016; Salehpour et al., 2012; Yang et al., 2016; Unfer et al., 2012; Aquino \& Nori, 2014; Artini et al., 2013).

Conventional treatments for PCOS, which include management of symptoms and clinical signs, have little effect on long-term complications such as cardiovascular disease and hyperinsulinemia. In recent years, complementary therapies including lifestyle changes, yoga, acupuncture, aromatherapy, homeopathy, weight loss, medicinal herbs, and vitamins have been used (Genazzani et al., 2004; Aquino \& Nori, 2014; Ratnakumari et al., 2018; Baillargeon \& Nestler, 2006).

Several studies have described an association between PCOS and insulin resistance. Central obesity has been described in $30-40 \%$ of women with PCOS, while hyperinsulinemia affects more than $80 \%$ of them. Insulin resistance increases significantly with obesity, which may disturb ovulation and increase androgen levels. Some treatments of PCOS with metformin, pioglitazone, and troglitazone have described improved ovarian function by managing hyperinsulinemia and insulin resistance. Increased sensitivity to gonadotropins due to increased insulin sensitivity may lead to spontaneous ovulation and pregnancy. Furthermore, treatments that decrease insulin resistance - such as protocols with metformin - may increase the fertility rates of women with PCOS (Genazzani et al., 2004; 2007; Motta, 2012; Naderpoor et al., 2015; De Leo et al., 2003; Steiber et al., 2004; Suvarna et al., 2016; Ou et al., 2017; Vanella et al., 2000; Pillich et al., 2005).

Carnitine is a quaternary amine synthesized in the body from amino acids lysine and methionine. In living cells, this chemical agent can transfer fatty acids from the cytosol to the mitochondria to produce energy from fatty acids. Carnitine is often used as a micronutrient and is divided into two types: L-carnitine (active form) and D-carnitine (inactive form). L-carnitine plays an important role in glucose metabolism and oxidative stress. L-carnitine can also stabilize the mitochondrial membranes and prevent cell apoptosis (Ringseis et al., 2012; Ismail et al., 2014; Fen$\mathrm{kci}$ et al., 2008). Some authors have looked into the role of carnitine in the treatment of insulin resistance and in the accumulation of acetyl coenzyme $A$. Insulin resistance has been linked to the occurrence of carnitine deficiency during chronic metabolic stress conditions such as diabetes mellitus type 2 and obesity. Recent studies have reported decreased levels of L-carnitine in patients with PCOS and apparently significant correlations between lower levels of L-carnitine and greater chances of individuals with PCOS developing hyperinsulinemia (Bacurau et al., 2003; Wächter et al., 2002; Karlic \& Lohninger, 2004; Jamilian et al., 2017).

This study aimed to assess the effects of L-carnitine on the clinical and laboratory parameters and metabolic profiles of individuals with PCOS.

\section{MATERIALS AND METHODS}

Eighty women of reproductive age diagnosed with PCOS based on the Rotterdam criteria (Rotterdam ESHRE/ASRM-Sponsored PCOS Consensus Workshop Group, 2004a,b) were enrolled in the study at the 
gynecology clinic of the Taleghani Hospital between 2017 and 2018. All participants gave written consent before joining the study. The Ethics Committee of the Shahid Beheshti University of Medical Sciences (SBMU) approved the study. Patients with adrenal deficiency or other endocrine conditions and individuals on hormone therapy in the six months prior to the study were excluded.

The women included in the study received L-carnitine $3 \mathrm{~g}$ daily (Pursinapharma, Iran) for three months. The patients were not instructed to follow a specific diet or introduce lifestyle changes. Blood samples were taken after overnight fasting at baseline and three months into the study to assess the levels of fasting glucose, insulin, triglycerides, high-density lipoprotein (HDL), low-density lipoprotein (LDL), free testosterone, dehydroepiandrosterone (DHEA), and the insulin resistance index (HOMA-IR). The patients were weighed before and after treatment and had their body mass index (BMI) calculated. The HOMA index was calculated as [baseline glucose] $x$ [baseline insulin]/22.5. Menstrual cycles and manifestations of hirsutism were also assessed.

Sample size was determined after consideration for type 1 statistical error $<5 \%$ and type 2 statistical error $<20 \%$. Results were shown as mean values plus or minus SD (Standard Deviation). Statistical analysis was performed using statistical software package SPSS 21.0 (SPSS Inc., Chicago, IL, USA). A $P$ value of 0.05 was considered significant.

\section{RESULTS}

Eighty patients with PCOS who met the inclusion criteria were enrolled in the study. Six participants were lost during the study.

Data are shown as mean values \pm SD. Table 1 provides a summary of baseline characteristics and results after three months of treatment. There were significant decreases in the levels of fasting glucose, insulin, triglycerides, LDL, and in the BMI and HOMA index. There was a significant increase in the level of HDL. Regular menstrual cycles were reported by $48.6 \%$ and $61.1 \%$ of the patients before and after treatment, respectively. No relevant side effects were reported during and after the treatment.

\section{DISCUSSION}

PCOS is a common disorder in women of reproductive age closely tied to insulin resistance, a condition associated with obesity, metabolic syndrome, gestational diabetes, type 2 diabetes, and cardiovascular disease. The management of PCOS may be challenging on account of the comorbidities associated with the disease. Recent studies have focused on the long-term complications of PCOS. Hormonal contraceptives, insulin-sensitizing drugs such as metformin, thiazolidinediones, myo-inositol, statins, orlistat, and $\mathrm{N}$-acetylcysteine have been prescribed to women with PCOS, but their usage is limited due to side effects.

L-carnitine supplementation has been recently used in obese patients to enhance the metabolic cascade. In the body, L-carnitine is produced in the liver and kidneys and stored in the musculoskeletal system, heart, brain, and sperm. L-carnitine supplementation is used to increase energy consumption and reduce lipids and weight (Vanella et al., 2000; Pillich et al., 2005; Ringseis et al., 2012; Ismail et al., 2014; Fenkci et al., 2008). L-carnitine plays an important role in glucose metabolism and oxidative stress. According to the literature, low serum levels of L-carnitine, even in non-obese women, may be associated with insulin resistance and hyperandrogenism (Salehpour et al., 2016; Celiket al., 2017; Samimi et al., 2016; Ismail et al., 2014).

This study looked into the effects of L-carnitine on the clinical and laboratory parameters of women with PCOS. The results showed a significant reduction in the BMI and serum levels of TG, LDL, FBS, and insulin, in addition to increased serum HDL levels. Improvement in menstrual cycle regularity was reported without drug-related side effects. According to the results, it appears that treatment with L-carnitine may have improved the hormonal and metabolic parameters of women with PCOS.

Ismail et al. (2014) reported that prescribing L-carnitine to clomiphene-resistant patients with PCOS improved the quality of ovulation, pregnancy rates, lipid profiles, and the BMI. It has been established that L-carnitine is safe and may be used to eliminate the long-term complications of PCOS. The long-term effects of L-carnitine for women with PCOS should be further evaluated. Studies with longer treatment cycles should be conducted to confirm the value of this therapy for women with PCOS at risk of metabolic syndrome and cardiovascular disorders.

Table 1. Patient characteristics and results

\begin{tabular}{|c|c|c|c|}
\hline & Pre treatment & Post treatment & $p$ value \\
\hline $\mathrm{FBS}(\mathrm{mg} / \mathrm{dl} \pm \mathrm{SD})$ & $92.90 \pm 11.83$ & $87.12 \pm 9.41$ & $<0.001^{*}$ \\
\hline Free testosterone $(\mathrm{ng} / \mathrm{ml} \pm \mathrm{SD})$ & $0.483 \pm 0.149$ & $0.467 \pm 0.144$ & 0.232 \\
\hline Insulin (mUI/I $\pm S D)$ & $23.60 \pm 4.29$ & $17.31 \pm 5.15$ & $<0.001^{*}$ \\
\hline DHEA (micromol/I $\pm S D$ ) & $1.454 \pm 0.190$ & $1.406 \pm 0.336$ & 0.252 \\
\hline Ferriman-Gallwey score & $6.62 \pm 2.17$ & $6.26 \pm 2.52$ & 0.062 \\
\hline $\mathrm{LDL}(\mathrm{mg} / \mathrm{dl} \pm \mathrm{SD})$ & $169.47 \pm 32.7$ & $152.47 \pm 32.19$ & $<0.001^{*}$ \\
\hline $\mathrm{HDL}(\mathrm{mg} / \mathrm{dl} \pm \mathrm{SD})$ & $35.62 \pm 5.39$ & $39.11 \pm 5.86$ & $<0.001^{*}$ \\
\hline Triglycerides (mg/dl \pm SD) & $202.03 \pm 45.31$ & $166.92 \pm 40.76$ & $<0.001 *$ \\
\hline BMI $\left(\mathrm{kg} / \mathrm{m}^{2} \pm \mathrm{SD}\right)$ & $28.28 \pm 2.6$ & $26.82 \pm 2.46$ & $<0.001 *$ \\
\hline Menstrual regularity (\%) & 48.6 & 61.1 & $<0.001^{*}$ \\
\hline HOMA-IR & $96.22 \pm 18.18$ & $67.04 \pm 22$ & $<0.001 *$ \\
\hline
\end{tabular}

* Statistically significant difference

FBS: Fasting blood sugar, DHEA: Dehydroepiandrosterone LDL: Low-density lipoprotein, HDL: High-density lipoprotein, BMI: Body mass index

$S D$ : standard deviation 


\section{CONCLUSION}

It appears that treatment with L-carnitine may decrease the risk of cardiovascular events by normalizing metabolic profiles and the BMI.

Acronyms:

1. FBS: Fasting blood sugar

2. BMI: Body mass index

3. HDL: High density lipoprotein

4. LDL: Low density lipoprotein

5. TG: Triglycerides

6. DHEA: Dehydroepiandrosterone

7. PCOS: Polycystic ovary syndrome

8. HOMA index: calculated as [baseline glucose] $x$ [baseline insulin]/22.5.

9. SD: Standard Deviation

\section{ACKNOWLEDGMENTS}

This article has been extracted from the thesis written by Dr. Bameni Moghaddam in the School of Medicine, Shahid Beheshti University of Medical Sciences.

\section{Conflicts of Interest Statement and Funding/ Support Statement}

The authors have no conflict of interest to declare.

\section{Corresponding author:}

Leila Nazari

Department of Obstetrics and Gynecology

IVF center

Taleghani Hospital

Velenjak St, Chamran Highway, Tehran, Iran.

Email: nazari@sbmu.ac.ir

\section{REFERENCES}

Aquino CI, Nori SL. Complementary therapy in polycystic ovary syndrome. Transl Med UniSa. 2014;9:56-65. PMID: 24809037

Artini PG, Di Berardino OM, Papini F, Genazzani AD, Simi G, Ruggiero M, Cela V. Endocrine and clinical effects of myo-inositol administration in policystyc ovary syndrome. A randomized study. Gynecol Endocrinol. 2013;29:375-9. PMID: 23336594 DOI: 10.3109/09513590.2012.743020

Bacurau RF, Navarro F, Bassit RA, Meneguello MO, Santos RV, Almeida AL, Costa Rosa LF. Does exercise training interfere with the effects of L-carnitine supplementation? Nutrition. 2003;19:337-41. PMID: 12679168

Baillargeon JP, Nestler JE. Commentary: polycystic ovary syndrome: a syndrome of ovarian hypersensitivity to insulin? J Clin Endocrinol Metab. 2006;91:22-4. PMID: 16263814 DOI: $10.1210 /$ jc.2005-1804

Banaszewska B, Pawelczyk L, Spaczynski RZ, Duleba AJ. Comparison of simvastatin and metformin in treatment of polycystic ovary syndrome: prospective randomized trial. J Clin Endocrinol Metab. 2009;94:4938-45. PMID: 19890022 DOI: $10.1210 /$ jc. $2009-1674$

Banaszewska B, Pawelczyk L, Spaczynski RZ, Duleba AJ. Effects of simvastatin and metformin on polycystic ovary syndrome after six months of treatment. J Clin Endocrinol Metab. 2011;96:3493-501. PMID: 21865358 DOI: 10.1210/jc.2011-0501
Celik F, Kose M, Yilmazer M, Köken GN, Arioz DT, Kanat Pektas M. Plasma L-carnitine levels of obese and nonobese polycystic ovary syndrome patients. J Obstet Gynaecol. 2017;37:476-9. PMID: 28141959 DOI: $10.1080 / 01443615.2016 .1264375$

De Leo V, la Marca A, Petraglia F. Insulin-lowering agents in the management of polycystic ovary syndrome. Endocr Rev. 2003;24:633-67. PMID: 14570747 DOI: $10.1210 /$ er.2002-0015

Fenkci SM, Fenkci V, Oztekin O, Rota S, Karagenc N. Serum total L-carnitine levels in non-obese women with polycystic ovary syndrome. Hum Reprod. 2008;23:1602-6. PMID: 18378560 DOI: $10.1093 /$ humrep/den109

Genazzani AD, Battaglia C, Malavasi B, Strucchi C, Tortolani F, Gamba O. Metformin administration modulates and restores luteinizing hormone spontaneous episodic secretion and ovarian function in nonobese patients with polycystic ovary syndrome. Fertil Steril. 2004;81:114-9. PMID: 14711553 DOI: $10.1016 /$ j.fertnstert.2003.05.020

Genazzani AD, Lanzoni C, Ricchieri F, Baraldi E, Casarosa $E$, Jasonni VM. Metformin administration is more effective when non-obese patients with polycystic ovary syndrome show both hyperandrogenism and hyperinsulinemia. Gynecol Endocrinol. 2007;23:146-52. PMID: 17454168 DOI: $10.1080 / 09513590701214398$

Ismail AM, Hamed AH, Saso S, Thabet HH. Adding L-carnitine to clomiphene resistant PCOS women improves the quality of ovulation and the pregnancy rate. A randomized clinical trial. Eur J Obstet Gynecol Reprod Biol. 2014;180:148-52. PMID: 25015747 DOI: 10.1016/j.ejogrb.2014.06.008

Jamilian $H$, Jamilian $M$, Samimi $M$, Afshar Ebrahimi $F_{\text {, }}$ Rahimi M, Bahmani F, Aghababayan S, Kouhi M, Shahabbaspour S, Asemi Z. Oral carnitine supplementation influences mental health parameters and biomarkers of oxidative stress in women with polycystic ovary syndrome: a randomized, double-blind, placebo-controlled trial. Gynecol Endocrinol. 2017;33:442-7. PMID: 28277138 DOI: $10.1080 / 09513590.2017 .1290071$

Karlic $\mathrm{H}$, Lohninger A. Supplementation of L-carnitine in athletes: does it make sense? Nutrition. 2004;20:709-15. PMID: 15212755 DOI: 10.1016/j.nut.2004.04.003

Kumar AN, Naidu JN, Satyanarayana U, Ramalingam K, Anitha M. Metabolic and Endocrine Characteristics of Indian Women with Polycystic Ovary Syndrome. Int J Fertil Steril. 2016;10:22-8. PMID: 27123196

Marshall JC, Dunaif A. Should all women with PCOS be treated for insulin resistance? Fertil Steril. 2012;97:18-22. PMID: 22192137 DOI: 10.1016/j.fertnstert.2011.11.036

Motta $A B$. The role of obesity in the development of polycystic ovary syndrome. Curr Pharm Des. 2012;18:248291. PMID: 22376149 DOI: 10.2174/13816128112092482

Naderpoor N, Shorakae S, Joham A, Boyle J, De Courten B, Teede $\mathrm{HJ}$. Obesity and polycystic ovary syndrome. Minerva Endocrinol. 2015;40:37-51. PMID: 17912153 
Ou HT, Chen PC, Wu MH. Effect of metformin by employing 2-hour postload insulin for measuring insulin resistance in Taiwanese women with polycystic ovary syndrome. J Formos Med Assoc. 2017;116:80-9. PMID: 27131512 DOI: 10.1016/j.jfma.2016.02.001

Pillich RT, Scarsella G, Risuleo G. Reduction of apoptosis through the mitochondrial pathway by the administration of acetyl-L-carnitine to mouse fibroblasts in culture. Exp Cell Res. 2005;306:1-8. PMID: 15878327 DOI: $10.1016 /$ j.yexcr.2005.01.019

Raja-Khan N, Stener-Victorin E, Wu X, Legro RS. The physiological basis of complementary and alternative medicines for polycystic ovary syndrome. Am J Physiol Endocrinol Metab. 2011;301:E1-E10. PMID: 21487075 DOI: 10.1152/ ajpendo.00667.2010

Ratnakumari ME, Manavalan N, Sathyanath D, Ayda YR, Reka K. Study to Evaluate the Changes in Polycystic Ovarian Morphology after Naturopathic and Yogic Interventions. Int J Yoga. 2018;11:139-47. PMID: 29755223 DOI: 10.4103/ijoy.IJOY_62_16

Ringseis R, Keller J, Eder K. Role of carnitine in the regulation of glucose homeostasis and insulin sensitivity: evidence from in vivo and in vitro studies with carnitine supplementation and carnitine deficiency. Eur J Nutr. 2012;51:1-18. PMID: 22134503 DOI: 10.1007/s00394-011-0284-2

Rotterdam ESHRE/ASRM-Sponsored PCOS Consensus Workshop Group. Revised 2003 consensus on diagnostic criteria and long-term health risks related to polycystic ovary syndrome.Fertil Steril. 2004a;81:19-25

Rotterdam ESHRE/ASRM-Sponsored PCOS consensus workshop group. Revised 2003 consensus on diagnostic criteria and long-term health risks related to polycystic ovary syndrome (PCOS). Hum Reprod. 2004b;19:41-7

Salehpour S, Sene AA, Saharkhiz N, Sohrabi MR, Moghimian F. N-Acetylcysteine as an adjuvant to clomiphene citrate for successful induction of ovulation in infertile patients with polycystic ovary syndrome. J Obstet Gynaecol Res. 2012;38:1182-6. PMID: 22540635 DOI: $10.1111 /$ j.14470756.2012.01844.X

Salehpour S, Nazari L, Hoseini S, Saharkhiz N, Ghazi F, Sohrabi MR. A Potential Therapeutic Role of Myoinositol in the Metabolic and Cardiovascular Profile of PCOS Iranian Women Aged between 30 and 40 Years. Int J Endocrinol. 2016;2016:7493147. PMID: 27648072 DOI: $10.1155 / 2016 / 7493147$
Samimi M, Jamilian M, Ebrahimi FA, Rahimi M, Tajbakhsh $B$, Asemi Z. Oral carnitine supplementation reduces body weight and insulin resistance in women with polycystic ovary syndrome: a randomized, double-blind, placebo-controlled trial. Clin Endocrinol (Oxf). 2016;84:851-7. PMID: 26666519 DOI: 10.1111/cen.13003

Steiber A, Kerner J, Hoppel CL. Carnitine: a nutritional, biosynthetic, and functional perspective. Mol Aspects Med. 2004;25:455-73. PMID: 15363636 DOI: 10.1016/j.mam.2004.06.006

Stener-Victorin E, Holm G, Janson PO, Gustafson D, Waerm $M$. Acupuncture and physical exercise for affective symptoms and health-related quality of life in polycystic ovary syndrome: secondary analysis from a randomized controlled trial. BMC Complement Altern Med. 2013;13:131. PMID: 23763822 DOI: 10.1186/1472-6882-13-131

Suvarna Y, Maity N, Kalra P, Shivamurthy MC. Comparison of efficacy of metformin and oral contraceptive combination of ethinyl estradiol and drospirenone in polycystic ovary syndrome. J Turk Ger Gynecol Assoc. 2016;17:6-9. PMID: 27026772 DOI: 10.5152/jtgga.2016.16129

Unfer V, Carlomagno G, Dante G, Facchinetti F. Effects of myo-inositol in women with PCOS: a systematic review of randomized controlled trials. Gynecol Endocrinol. 2012;28:509-15. PMID: 22296306 DOI: $10.3109 / 09513590.2011 .650660$

Vanella A, Russo A, Acquaviva R, Campisi A, Di Giacomo C, Sorrenti $\mathrm{V}$, Barcellona ML. L-propionyl-carnitine as superoxide scavenger, antioxidant, and DNA cleavage protector. Cell Biol Toxicol. 2000;16:99-104. PMID: 10917565 DOI: 10.1023/A: 1007638025856

Wächter S, Vogt M, Kreis R, Boesch C, Bigler P, Hoppeler $\mathrm{H}$, Krähenbühl S. Long-term administration of L-carnitine to humans: effect on skeletal muscle carnitine content and physical performance. Clin Chim Acta. 2002;318:51-61. PMID: 11880112 DOI: 10.1016/S0009-8981(01)00804-X

Yang B, Sun ZJ, Chen B, Zhang J, Zhao H, Li CW, Cao YK, Cao F. Statin ameliorates endothelial dysfunction and insulin resistance in Tibet women with polycystic ovary syndrome. Eur Rev Med Pharmacol Sci. 2016;20:1185-91. PMID: 27049276 